\title{
КЛИНИЧЕСКИЙ ПРИМЕР ВОССТАНОВЛЕНИЯ ПРОХОДИМОСТИ ПЕЧЕНОЧНОЙ ВЕНЫ И ПОРТОСИСТЕМНОГО ШУНТА ЧЕРЕЗ 10 ЛЕТ ПОСЛЕ ТІРS
}

\author{
М. Ш. Цициашвили', В. Н. Шиповскийํ, \\ 1 Российский национальный исследовательский университет имени Н. И. Пирогова, Москва, Россия \\ 2 Городская клиническая больница имени Д. Д. Плетнева, Москва, Россия

\begin{abstract}
Одним из немногих доступных методов борьбы с осложнениями портальной гипертензии у пациентов с хроническими дифффузными заболеваниями печени является трансъюгулярное портосистемное шунтирование. С течением времени имеется тенденция к развитию диссункции внутрипеченочного шунта вследствие нарушения его проходимости, что вновь приводит к развитию осложнений портальной гипертензии. Описанный клинический случай демонстрирует возможности внутрипеченочного шунтирования, а также повторных эндоваскулярных вмешательств при диссункции шунта, позволяющих значительно продлить жизнь пациента и сохранить ее качество.
\end{abstract}

Ключевые слова: цирроз печени, портальная гипертензия, трансъюгулярное внутрипеченочное портосистемное шунтирование, диссункция, реинтервенция

Информация о вкладе авторов: А. С. Челяпин, А. Б. Гусейнов - концепция и дизайн исследования, сбор и обработка материала; А. С. Челяпин статистическая обработка данных; М. Ш. Цициашвили, В. Н. Шиповский, Д. В. Монахов, А. С. Челяпин, А. Б. Гусейнов - написание текста; М. Ш. Цициашвили - редактирование.

Соблюдение этических стандартов: работа одобрена этическим комитетом РНИМУ им. Н. И. Пирогова (протокол № 170 от 18 декабря 2017 г.) Все участники исследования подписали информированное согласие на участие в исследовании

$\triangle$ Для корреспонденции: Александр Сергеевич Челяпин

ул. Островитянова, д. 1, г. Москва, 117997; ChelyapinAleks@yandex.ru

Статья получена: 24.10.2019 Статья принята к печати: 08.11.2019 Опубликована онлайн: 16.11.2019

DOI: $10.24075 /$ vrgmu.2019.074

\section{RE-ESTABLISHING THE PATENCY OF THE HEPATIC VEIN AND THE PORTOSYSTEMIC SHUNT 10 YEARS AFTER THE TIPS PROCEDURE: A CLINICAL CASE}

Tsitsiashvili MSh ${ }^{1}$, Shipovskiy VN ${ }^{1,2}$, Monakhov DV ${ }^{1,2}$, Chelyapin AS $1,2 \varangle$, Huseynov AB ${ }^{1}$

${ }^{1}$ Pirogov Russian National Research Medical University, Moscow, Russia

2 Pletnev City Clinical Hospital, Moscow, Russia

Transjugular intrahepatic portosystemic shunt is one of the few available options for treating complications of portal hypertension in patients with chronic diffuse liver diseases. Over time, shunt patency can become compromised, causing a recurrence of such complications. The clinical case presented below demonstrates the potential of TIPS and illustrates the use of an endovascular reintervention for re-establishing shunt patency, which improves the life expectancy of patients, as well as their quality of life.

Keywords: liver cirrhosis, portal hypertension, transjugular intrahepatic portosystemic shunt, dysfunction, reintervention

Author contribution: Chelyapin AS, Huseynov AB - study conception and design, data acquisition and processing; Chelyapin AS — statistical analysis; Tsitsiashvili MSh, Shipovskiy VN, Monakhov DV, Chelyapin AS, Huseynov AB — manuscript draft; Tsitsiashvili MSh — manuscript revision.

Compliance with ethical standards: the study was approved by the Ethics Committee of Pirogov Russian National Research Medical University (Protocol 170 dated December 18, 2017). Informed consent was obtained from all study participants.

$\triangle$ Correspondence should be addressed: Alexander Chelyapin

Ostrovityanova, 1, Moscow, 117997; ChelyapinAleks@yandex.ru

Received: 24.10.2019 Accepted: 08.11.2019 Published online: 16.11.2019

DOI: $10.24075 /$ brsmu.2019.074

Наиболее серьезное осложнение синдрома портальной гипертензии - кровотечение из варикозно-расширенных вен пищевода и желудка (ВРВП и ВРВЖ). Летальность при первом эпизоде геморрагий достигает 50\%, а при рецидиве кровотечения - 70\% [1, 2]. Для снижения летальности в России разработан специальный алгоритм, изложенный В «клинических рекомендациях по лечению кровотечений из варикозно-расширенных вен пищевода и желудка» [3]. Согласно алгоритму, после выполнения экстренной эзофагогастродуоденоскопии (ЭГДС) приоритетным эндоскопическим методом является лигирование вен пищевода, применение которого позволяет добиться гемостаза у 80\% пациентов с продолжающимся кровотечением [3]. Однако данная методика наиболее эффективна в качестве профилактики первого эпизода кровотечения и при этом не влияет на давление в воротной вене. Повторное появление ВРВП и рецидив кровотечения в отдаленном послеоперационном периоде наблюдают в 50-60\% случаях [4]. В связи с этим для пациентов с рецидивирующими кровотечениями предложены такие хирургические вмешательства, как операции портосистемного шунтирования, непосредственно снижающие давление в портальной системе, а также операции азигопортального разобщения. Среди операций портосистемного шунтирования у пациентов с циррозом печени класса В и С методом выбора является операция трансъюгулярного внутрипеченочного портосистемного шунтирования (TIPS). В настоящее время нашей клиникой накоплен опыт выполнения 226 подобных операций.

Актуальная проблема внутрипеченочного шунтирования нарушение проходимости стента, приводящее к рецидиву осложнений портальной гипертензии [5-8]. Причинами нарушений проходимости шунта могут быть тромбоз и псевдоинтимальная гиперплазия TIPS, гиперплазия 
интимы печеночной вены, сдавление стента тканью печени высокой плотности, миграция и фрагментация стента. Средняя продолжительность работы шунта при использовании стент-графта, по нашим данным, составляет 3-5 лет, в единичных случаях — до 10 лет.

На сегодняшний день разработаны различные виды эндоваскулярных вмешательств, направленных на восстановление проходимости шунта. Применение подобных операций позволяет увеличить срок функционирования внутрипеченочного шунта, снизить риск развития осложнений портальной гипертензии, тем самым продлить жизнь пациентов с терминальными стадиями диффузных хронических заболеваний печени.

В изложенном клиническом случае мы приводим опыт выполнения повторной операции после TIPS через 10 лет после имплантации стента в паренхиму печени.

\section{Описание клинического случая}

Впервые диагноз цирроза печени (ЦП), осложненный синдромом портальной гипертензии, пациенту К. (возраст 40 лет) был установлен в 2006 г. в ГУЗ «Центральном научно-исследовательском институте гастроэнтерологии ДЗ г. Москвы». 10 декабря 2007 г. пациент поступил в 4-е хирургическое отделение ГКБ № 57 ДЗ г. Москвы в срочном порядке с диагнозом: цирроз печени, класс В по Чайлду-Пью, осложненный синдромом портальной гипертензии и состоявшимся кровотечением из ВРВП III степени.

При поступлении состояние пациента средней тяжести. Кожные покровы бледные, склеры субиктеричные. В легких дыхание везикулярное, проводится во все отделы. ЧДД - 14 в минуту. Тоны сердца приглушены. АД - 110/70 в минуту, ЧСС - 96 в минуту. Живот мягкий, безболезненный во всех отделах. Печень выступает из-под края реберной дуги на 3 см. Патологии со стороны мочевыделительной системы не выявлено.

По результатам инструментальных методов диагностики у больного диагностированы следующие признаки портальной гипертензии: дилатация воротной (14 мм) и селезеночной (9 мм) вен, гепатоспленомегалия, ВРВП III степени. При обследовании выявлена постгеморрагическая анемия легкой степени тяжести: снижение уровня гемоглобина до 96 г/л, снижение количества эритроцитов до $3,89-10^{12} / л$. Отмечены тромбоцитопения $\left(102 \times 10^{9}\right.$ л) и гипербилирубинемия за счет непрямой фракции (34 мкмоль/л). Изменений в коагулограмме не обнаружено.

На вторые сутки от момента поступления у пациента рецидивировало желудочно-кишечное кровотечение. Диагноз продолжающегося кровотечения из ВРВП ІІІ степени был подтвержден при ЭГДС. В дальнейшем был выполнен гемостаз с помощью зонда-обтуратора и лечение продолжили в условиях отделения реанимации. При контрольной ЭГДС в средней и нижней третях пищевода выявили варикозно-расширенные венозные стволы диметром до 7-9 мм, с синюшного цвета слизистой над ними, покрытой налетом солянокислого гематина. Для снижения риска рецидива кровотечения пациенту первым этапом 17 декабря 2007 г. выполнили эндоскопическое лигирование ВРВП, наложив 20 лигатур. Вторым этапом 19 декабря 2007 г. провели трансъюгулярное внутрипеченочное портосистемное шунтирование стент-графтом Viatorr TIPS Endoprosthesis (Gore; USA). Интраоперационно было отмечено снижение давления в воротной вене с 520 мм водн. ст. до 300 мм водн. Ст.

Послеоперационный период протекал гладко; 22 декабря 2007 г. была выполнена контрольная ультразвуковая допплерограсрия, по данным которой выявлен магистральный кровоток по стенту со скоростью $85 \mathrm{~cm} / \mathrm{c}$. На фоне проводимой консервативной терапии состояние пациента стабилизировалось, 25 декабря 2007 г. пациент был выписан в удовлетворительном состоянии.

В дальнейшем в течение девяти лет пациент каждые полгода госпитализировался в нашу клинику для проведения гепатотропной терапии и динамического наблюдения. За данный период наблюдения (9 лет) клинических проявлений осложнений портальной гипертензии не было.

При очередной контрольной госпитализации (декабрь 2017 г.) по данным ультразвукового дуплексного сканирования (УЗДС) отмечено увеличение скорости кровотока в проксимальной части стента, снижение скорости кровотока в дистальной части, заподозрен стеноз правой печеночной вены (рис. 1). При ЭГДС выявлены ВРВП III степени, свидетельствующие о нарастании давления в системе воротной вены.

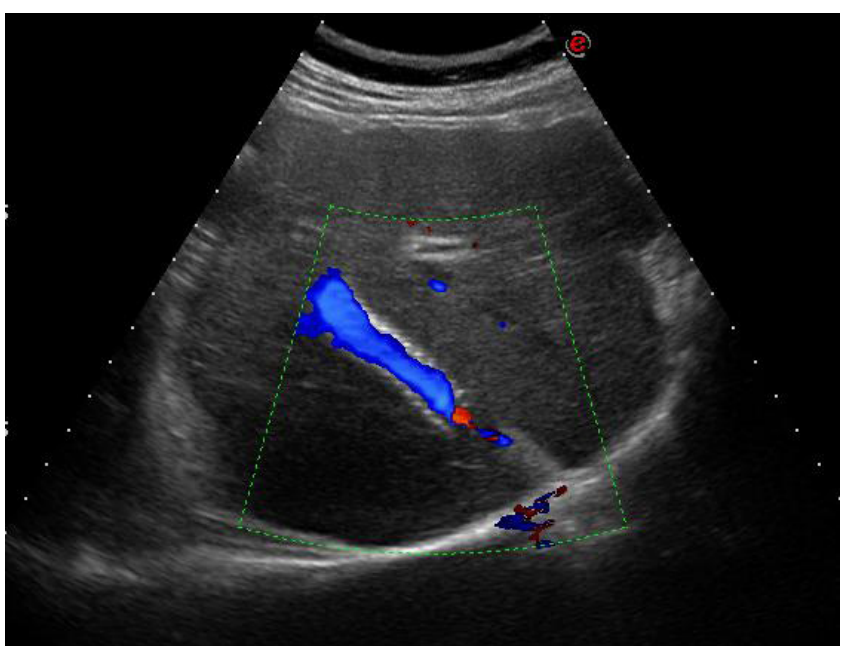

Рис. 1. Больной К. Цирроз печени, класс В, после TIPS. Стеноз проксимальной части TIPS. Скорость кровотока $37 \mathrm{~cm} / \mathrm{c}$

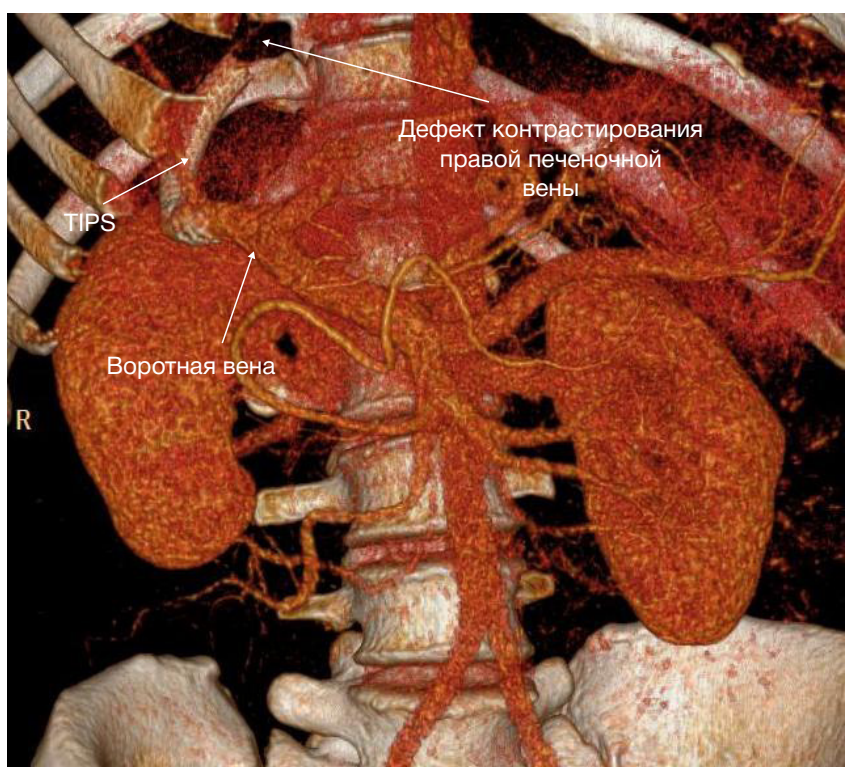

Рис. 2. КТ-ангиография при гипереплазии интимы правой печеночной вень 
С целью дальнейшего дообследования и верификации диагноза больному выполнили компьютерную томографию органов брюшной полости с внутривенным контрастированием. По данным исследования диагноз стеноза правой печеночной вены был подтвержден (рис. 2).

Учитывая признаки стеноза правой печеночной вены - изменение гемодинамических показателей во внутрипеченочном шунте, в портальной системе (снижение скорости в воротной вене), увеличение степени ВРВП - было принято решение о необходимости восстановления проходимости TIPS.

После выполнения портографии и визуализации стеноза принято решение о его нивелировании при помощи стентирования стент-в-стент (рис. 3 и 4).

При контрольной портографии после реинтервенции выявлено, что трансъюгулярный внутрипеченочный шунт полностью проходим - стеноз ликвидирован (рис. 5).

Послеоперационный период протекал гладко, при выполнении УЗДС портального кровотока выявлен магистральный кровоток во внутрипеченочном шунте со скоростью 112 см/с, проведен курс гепатотропной терапии, пациент был выписан в удовлетворительном состоянии.

В течение последующих двух лет пациент проходил наблюдение в нашей клинике, последняя госпитализация состоялась в августе 2019 г., осложнений портальной гипертензии за данный период наблюдения не выявлено.

\section{Обсуждение клинического случая}

Анализируя литературные данные и наш собственный опыт, мы пришли к выводу, что оптимально в качестве внутрипеченочного шунта при TIPS использовать стент-графрты. Применение стент-графтов позволяет не только уменьшить риск развития тромбоза шунта в раннем послеоперационном периоде, но и снизить частоту дисфункции TIPS в позднем послеоперационном периоде [8, 9].

Несмотря на все выше перечисленные преимущества при использовании стент-графтов, с течением времени может возникнуть нарушение проходимости внутрипеченочного шунта в результате стеноза, обусловленного гиперплазией интимы правой печеночной вены. Для снижения риска

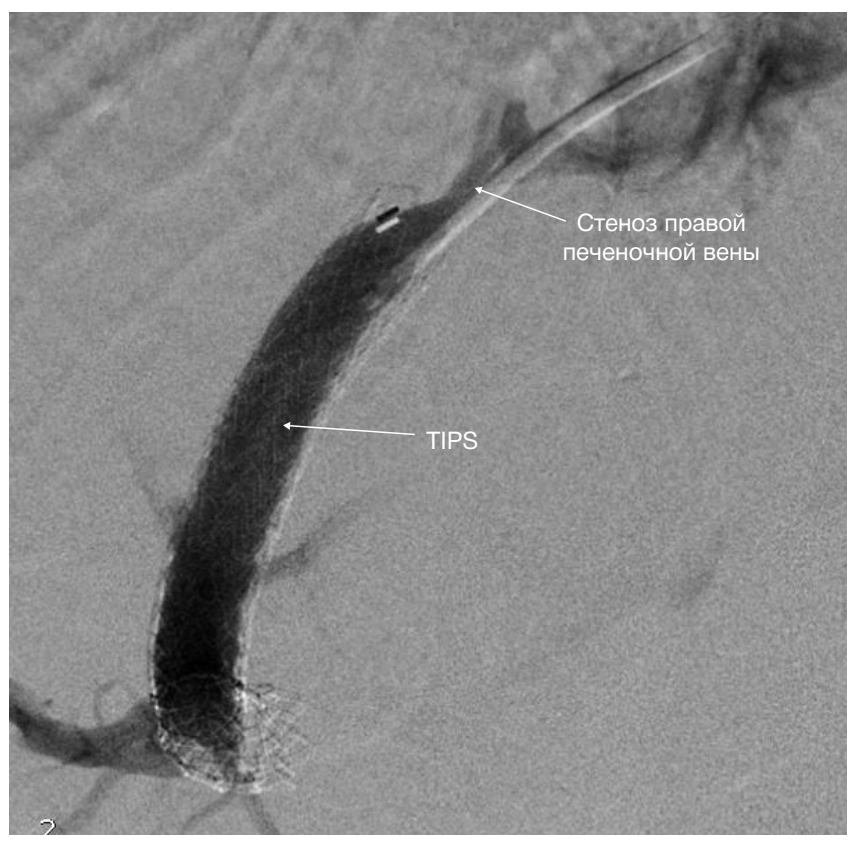

Рис. 3. Трансъюгулярная портография пациента К
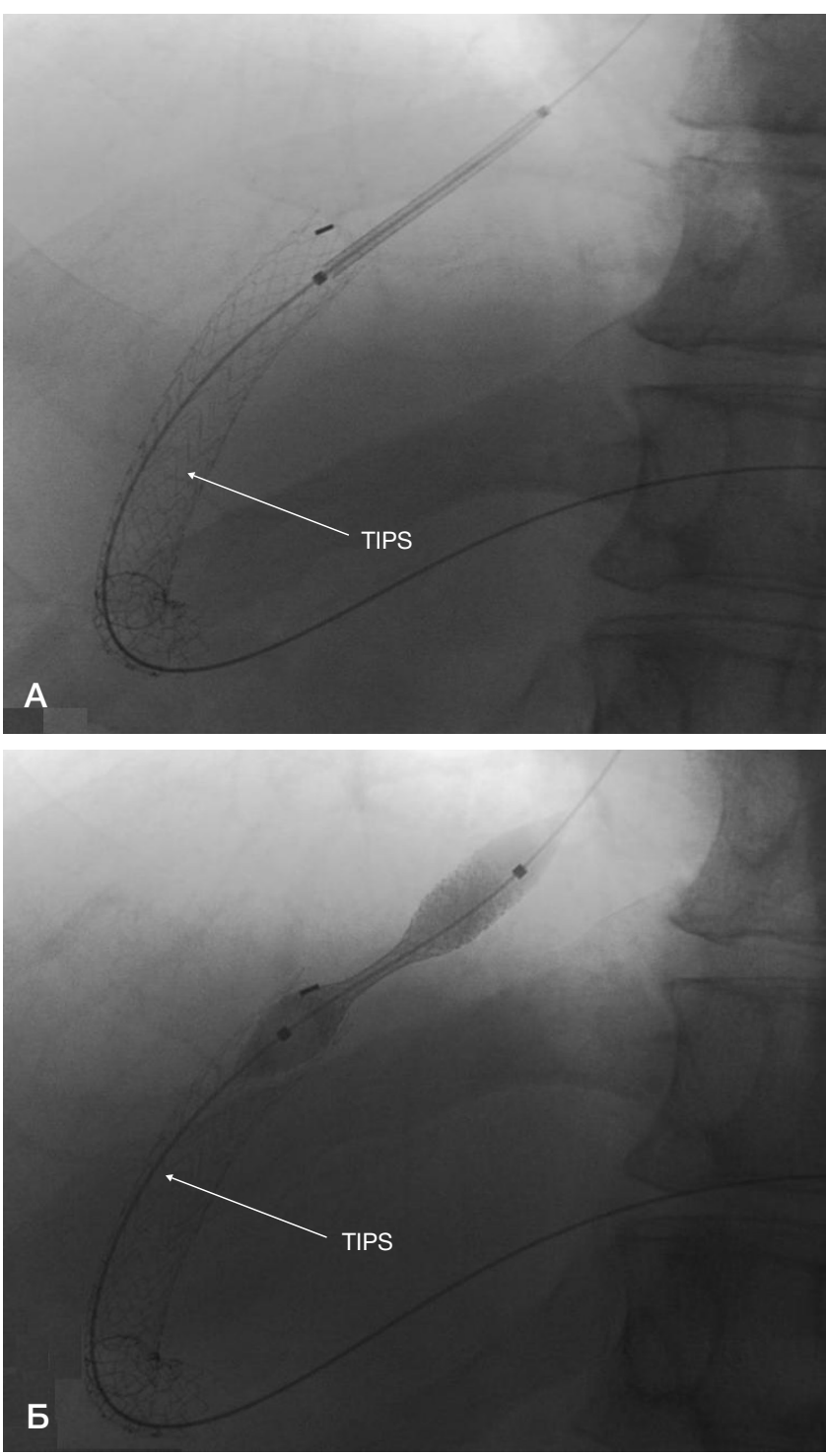

Рис. 4. Трансъюгулярная портография пациента К. Этапы стентирования стент-в-стент. А. Имплантация стента. Б. Раскрытие стента

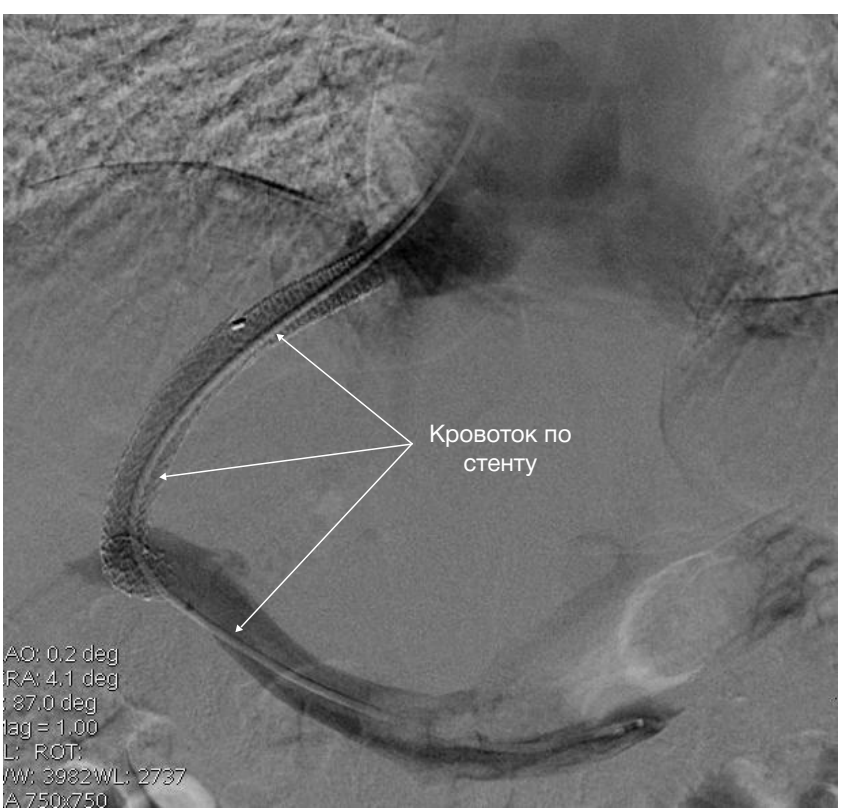

Рис. 5. Трансъюгулярная портограсия пациента К. после реинтервенции в объеме стетирования стент-в-стент 
развития данного состояния необходимо оптимальное позиционирование стента в устье правой печеночной вены $[10,11]$.

Как демонстрирует описанный клинический случай, реинтервенции после TIPS, включая стентирование правой печеной вены, позволяют не только продлить жизнь пациентов без развития осложнений портальной гипертензии, но и сохранить ее качество.

\section{Литература}

1. Elsebaey MA, et al. Endoscopic injection sclerotherapy versus $\mathrm{N}$-Butyl-2 Cyanoacrylate injection in the management of actively bleeding esophageal varices: a randomized controlled trial. BMC gastroenterology. 2019; 19 (1): 23.

2. Shi L, et al. Favorable Effects of Endoscopic Ligation Combined with Drugs on Rebleeding and Mortality in Cirrhotic Patients: A Network Meta-Analysis. Digestive Diseases. 2018; 36 (2): 136-49.

3. Анисимов А. Ю., Верткин А. Л., Девятов А. В., Дзидзава И. И., Жигалова С. Б., Затевахин И. И., и др. Клинические рекомендации по лечению кровотечений из варикознорасширенных вен пищевода и желудка. Доступно по ссылке: http://xn----9sbdbejx7bdduahou3a5d.xn--p1ai/stranica-pravlenija/ unkr/urgentnaja-abdominalnaja-hirurgija/klinicheskie-rekomendaci-polecheniyu-krovotechenii-iz-varikozno-rasshirenyh-ven-pischevoda-izheludka.html.

4. Masalaite L, Valantinas J, Stanaitis J. Endoscopic ultrasound findings predict the recurrence of esophageal varices after endoscopic band ligation: a prospective cohort study. Scandinavian journal of gastroenterology. 2015; 50 (11): 1322-30.

5. Bureau C, et al. Transjugular intrahepatic portosystemic shunts with covered stents increase transplant-free survival of patients

\section{References}

1. Elsebaey MA, et al. Endoscopic injection sclerotherapy versus $\mathrm{N}$-Butyl-2 Cyanoacrylate injection in the management of actively bleeding esophageal varices: a randomized controlled trial. BMC gastroenterology. 2019; 19 (1): 23.

2. Shi L, et al. Favorable Effects of Endoscopic Ligation Combined with Drugs on Rebleeding and Mortality in Cirrhotic Patients: A Network Meta-Analysis. Digestive Diseases. 2018; 36 (2): 136-49.

3. Anisimov AYu, Vertkin AL, Devyatov AV, Dzidzava II, Zhigalova SB, Zatevakhin II, et al. Clinical guidelines on treatment of esophageal and stomach variceal bleeding. Available from: http://xn---9sbdbejx7bdduahou3a5d.xn--p1ai/stranica-pravlenija/unkr/ urgentnaja-abdominalnaja-hirurgija/klinicheskie-rekomendaci-polecheniyu-krovotechenii-iz-varikozno-rasshirenyh-ven-pischevodai-zheludka.html.

4. Masalaite L, Valantinas J, Stanaitis J. Endoscopic ultrasound findings predict the recurrence of esophageal varices after endoscopic band ligation: a prospective cohort study. Scandinavian journal of gastroenterology. 2015; 50 (11): 1322-30

5. Bureau C, et al. Transjugular intrahepatic portosystemic shunts with covered stents increase transplant-free survival of patients with

\section{ВЫВОДЫ}

Восстановление проходимости внутрипеченочного шунта при его дисфункции играет важную роль, поскольку длительно функционирующий TIPS дает возможность пациенту достаточно долго находиться в листе ожидания трансплантации печени без угрозы возникновения кровотечения.

with cirrhosis and recurrent ascites. Gastroenterology. 2017; 152 (1): 157-63.

6. LV Y, Han G, Fan D. Thrombosis after transjugular intrahepatic portosystemic shunt: an ominous sign? AME Medical Journal. 2017; 2 (40).

7. $\mathrm{Li} \mathrm{YH}$, et al. Long-term shunt patency and overall survival of transjugular intrahepatic portosystemic shunt placement using covered stents with bare stents versus covered stents alone // Clinical radiology. 2018; 73 (6): 580-7.

8. Saad WE. A. Portal Hypertension: Imaging, Diagnosis, and Endovascular Management. Thieme, 2017.

9. Luo, Xue-Feng, et al. Stent-Grafts for the Treatment of TIPS Dysfunction: Fluency Stent vs Wallgraft Stent. World Journal of Gastroenterology. 2013; 19 (30): 5000-5.

10. Wang $Q$, et al. Eight millimetre covered TIPS does not compromise shunt function but reduces hepatic encephalopathy in preventing variceal rebleeding. Journal of hepatology. 2017; 67 (3): 508-16.

11. Suhocki PV, et al. Transjugular intrahepatic portosystemic shunt complications: prevention and management // Seminars in interventional radiology. Thieme Medical Publishers. 2015; 32 (2): 123-32.

cirrhosis and recurrent ascites. Gastroenterology. 2017; 152 (1): 157-63.

6. Lv Y, Han G, Fan D. Thrombosis after transjugular intrahepatic portosystemic shunt: an ominous sign? AME Medical Journal. 2017; 2 (40).

7. $\mathrm{Li} \mathrm{YH}$, et al. Long-term shunt patency and overall survival of transjugular intrahepatic portosystemic shunt placement using covered stents with bare stents versus covered stents alone // Clinical radiology. 2018; 73 (6): 580-7.

8. Saad WE. A. Portal Hypertension: Imaging, Diagnosis, and Endovascular Management. Thieme, 2017.

9. Luo, Xue-Feng, et al. Stent-Grafts for the Treatment of TIPS Dysfunction: Fluency Stent vs Wallgraft Stent. World Journal of Gastroenterology. 2013; 19 (30): 5000-5.

10. Wang $Q$, et al. Eight millimetre covered TIPS does not compromise shunt function but reduces hepatic encephalopathy in preventing variceal rebleeding. Journal of hepatology. 2017; 67 (3): 508-16.

11. Suhocki PV, et al. Transjugular intrahepatic portosystemic shunt complications: prevention and management // Seminars in interventional radiology. Thieme Medical Publishers. 2015; 32 (2): 123-32. 